\title{
Three-year experience with combined treatment with alendronate and alfacalcidol in Japanese patients with severe bone loss and osteoporotic fracture
}

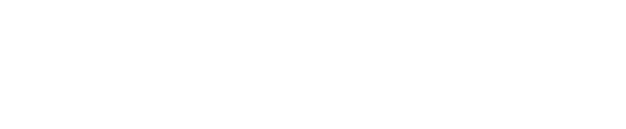

Jun Iwamoto'

Yoshihiro Sato ${ }^{2}$

Mitsuyoshi Uzawa ${ }^{3}$

Tsuyoshi Takeda'

Hideo Matsumoto'

'Institute for Integrated Sports

Medicine, Keio University School of

Medicine, Tokyo, Japan; ${ }^{2}$ Department of Neurology, Mitate Hospital,

Fukuoka, Japan; ${ }^{3}$ Department

of Orthopaedic Surgery, Keiyu

Orthopaedic Hospital, Gunma, Japan
Correspondence: Jun Iwamoto Institute for Integrated Sports Medicine, Keio University School of Medicine, 35 Shinanomachi, Shinjuku-ku,

Tokyo 160-8582, Japan

Tel +8133353 I2II

Fax $+8 I 333529467$

Email jiwamoto@a8.keio.jp.
Purpose: Combined treatment with alendronate and alfacalcidol is more useful to increase bone mineral density (BMD) than alendronate or alfacalcidol alone. A retrospective study was conducted to investigate the 3-year outcome of combined treatment with alendronate and alfacalcidol in patients with severe bone loss (BMD $\leq 50 \%$ of the young adult mean) and osteoporotic fracture.

Methods: Thirty-four patients (six men and 28 postmenopausal women) with primary or secondary osteoporosis who had been treated with alendronate and alfacalcidol for more than 3 years were analyzed. The lumbar spine or total hip BMD and bone turnover markers were monitored, and the incidence of osteoporotic fractures was assessed.

Results: The urinary level of cross-linked N-terminal telopeptides of type I collagen and serum level of alkaline phosphatase significantly decreased $(-42.5 \%$ at 3 months and $-18.9 \%$ at 3 years), and the lumbar spine BMD, but not the total hip BMD, significantly increased ( $14.8 \%$ at 3 years), compared with the baseline values. However, the incidence of vertebral and nonvertebral fractures was $26.5 \%$ and $2.9 \%$, respectively, suggesting a high incidence of vertebral fractures

Conclusion: The results of the present study suggest that combined treatment with alendronate and alfacalcidol may be useful to reduce bone turnover and increase the lumbar spine BMD in patients with severe bone loss and osteoporotic fracture. However, its efficacy against vertebral fractures appears not to be sufficient. Thus, anabolic agents such as teriparatide should be taken into consideration as first-line drugs in patients with severe osteoporosis.

Keywords: osteoporosis, fragility fracture, bone mineral density, bone turnover

\section{Introduction}

Alendronate (ALN) is widely used for the treatment of postmenopausal, glucocorticoidinduced, and male osteoporosis. The Fracture Intervention Trial demonstrated the efficacy of ALN against vertebral, nonvertebral, hip, and wrist fractures in postmenopausal women with osteoporosis. ${ }^{1,2}$ Furthermore, a recent systematic review analyzing 11 randomized controlled trials (RCTs) representing 12,068 women confirmed both clinically important and statistically significant reductions in vertebral, nonvertebral, hip, and wrist fractures for secondary prevention (gold-level evidence). ${ }^{3}$ ALN has also been reported to reduce the incidence of vertebral fractures in patients treated with glucocorticoid or patients with male osteoporosis..$^{4,5}$ 
The incidence of vertebral fractures is higher and that of hip fractures is lower among elderly Japanese people than among elderly Western people. ${ }^{6}$ ALN is regarded as a firstline drug for the treatment of postmenopausal osteoporosis in Japan, because RCTs in postmenopausal Japanese women with osteoporosis revealed that ALN treatment (1-3 years) suppressed bone turnover, increased the bone mineral density (BMD), and reduced the incidence of vertebral fractures. ${ }^{7-10}$ RCTs in Japanese patients treated with glucocorticoid showed that ALN treatment (1-2 years) sustained or increased the lumbar spine BMD. ${ }^{11-13}$ Furthermore, a RCT in Japanese men with osteoporosis or osteopenia and clinical risk factors of fractures confirmed that ALN treatment (1 year) increased the total hip BMD. ${ }^{14}$

In Japan, patients are diagnosed as having osteoporosis according to the Japanese diagnostic criteria. ${ }^{15,16}$ Namely, patients with a BMD $<70 \%$ of young adult mean (YAM) or $70 \%-80 \%$ of YAM along with a history of osteoporotic fractures were diagnosed as having osteoporosis. We have confirmed the long-term (4-7 years) efficacy and safety of ALN treatment in postmenopausal Japanese women or men with osteoporosis or osteopenia and clinical risk factors of fractures. ${ }^{17-19} \mathrm{In}$ our clinical practice, however, patients with severe bone loss (BMD $\leq 50 \%$ of YAM) are not common, and the effects of ALN treatment on BMD, bone turnover markers, and fracture incidence remain to be established in such patients.

The AAC-Trial demonstrated that combined treatment with ALN and alfacalcidol (ALF) exhibited superiority in terms of BMD, overall fractures, rate of falls, and back pain over either ALN in combination with plain vitamin D or ALF alone in established postmenopausal or male osteoporosis. ${ }^{20} \mathrm{An}$ RCT conducted by Felsenberg et $\mathrm{al}^{21}$ revealed that ALF increased the efficacy of ALN treatment in osteopenic/osteoporotic postmenopausal women on spine BMD, cortical and trabecular BMD of the tibia, and bending stiffness of the tibia. This line of evidence suggests the beneficial effect of combined treatment with ALN and ALF in patients with osteoporosis. A retrospective study was conducted to investigate the 3-year outcome of combined treatment with ALN and ALF in patients with severe bone loss (BMD $\leq 50 \%$ of YAM) and osteoporotic fracture. The incidences of osteoporotic fractures, including morphometric vertebral fractures and clinical vertebral and nonvertebral (hip, distal radius, and proximal humerus) fractures, were also assessed.

\section{Subjects and methods Subjects}

Thirty-four Japanese patients who had been treated with ALN and ALF for more than 3 years were recruited at the outpatient clinic of Keiyu Orthopaedic Hospital (Gunma, Japan) during the 3-month period between January 4 and April 4, 2011. The inclusion criteria were primary or secondary osteoporosis, patients with severe bone loss (BMD $\leq 50 \%$ of YAM) and osteoporotic vertebral, hip, distal radius, or proximal humerus fracture at the start of treatment. The exclusion criteria were histories of reflux esophagitis, gastric or duodenal ulcer, and renal failure.

The assessment prior to combined treatment with ALN and ALF included a medical history, physical examination, plain X-rays of the thoracic and lumbar spine, lumbar spine or total hip BMD measurement, and blood and urinary biochemical tests including serum calcium, phosphorus, and alkaline phosphatase (ALP), and urinary cross-linked $\mathrm{N}$-terminal telopeptides of type I collagen (NTX). The serum calcium, phosphorus, and ALP levels were measured using the standard laboratory technique, and the urinary NTX levels were measured using enzyme-linked immunosorbent assay. The lumbar spine or total hip BMD was measured using dualenergy X-ray absorptiometry (DXA) as described below.

The patients had been treated with ALN (5 mg daily or $35 \mathrm{mg}$ weekly) plus ALF ( $1 \mu \mathrm{g}$ daily). The doses indicated in the parentheses above are the doses used in Japan for the treatment of postmenopausal women with osteoporosis and have been recognized as being safe and effective..$^{7-10,22}$ Daily ALN was available throughout the study period, but weekly ALN only became available after October 2006. All the patients had been treated with daily ALN and then switched to weekly ALN just after October 2006. The effects of daily and weekly ALN on the BMD and bone turnover markers as well as the incidence of side effects were reported to be similar in postmenopausal Japanese women with osteoporosis. ${ }^{10}$

The urinary levels of NTX were measured at 3 months after the start of treatment, based on the results of our previous study. ${ }^{23}$ A urinary NTX measurement performed 3 months after the start of ALN treatment provides important information and is sufficient to monitor the effects of treatment for osteoporosis. ${ }^{23}$ The serum levels of calcium, phosphorus, and ALP and the lumbar spine or total hip BMD were measured every 6 months after the start of treatment. The outcome of combined treatment with ALN and ALF for 3 years was evaluated. The present study was approved by the Ethics Committee of Keiyu Orthopaedic Hospital.

\section{Assessment of morphometric vertebral fractures}

Plain lateral X-ray films of the thoracic and lumbar spine were obtained at baseline to detect evidence of morphometric 
vertebral fractures. According to the Japanese criteria, a vertebral fracture was defined according to the vertebral height on lateral X-ray films. ${ }^{15,16}$ Briefly, the vertebral height was measured at the anterior (A), central (C), and posterior (P) parts of the vertebral body, and the presence of a vertebral fracture was confirmed when (1) a reduction in the vertebral height of more than $20 \%$ (A, C, and P) compared with the height of the adjacent vertebrae was observed, (2) the $\mathrm{C} / \mathrm{A}$ or $\mathrm{C} / \mathrm{P}$ was less than 0.8 , or ( 3 ) the $\mathrm{A} / \mathrm{P}$ was less than 0.75 . The assessment for vertebral fractures was performed at the T4-L4 level.

\section{Assessment of clinical vertebral and nonvertebral fractures}

Low-traumatic osteoporotic clinical fractures were assessed. Clinical vertebral fractures were determined based on clinical symptoms and radiographs or magnetic resonance images of the lumbar and thoracic spine. Nonvertebral fractures in terms of osteoporotic fractures at the distal radius, proximal humerus, and hip, were determined based on clinical symptoms and radiographs of the wrist, shoulder, and hip joints, respectively.

\section{Measurement of lumbar spine or total hip BMD}

The BMD of the lumbar spine or total hip in the anteroposterior view was measured using DXA with a Hologic QDR 1500W apparatus (Hologic Inc, Bedford, MA). The coefficient of variation of five measurements with repositioning within 72 hours each time was less than $1.2 \%$ in three persons. Primarily, the lumbar spine (L1-L4) was chosen as a skeletal site for BMD measurement. However, the first lumbar vertebra (L1) was omitted for patients whose lumbar spine radiographs showed callus formation after L1 vertebral fracture. In this case, the lumbar spine (L2-L4) BMD was adopted. The total hip BMD was measured for patients whose lumbar spine radiographs showed severe spondylosis, severe scoliosis, callus formation after L2, L3, or L4 vertebral fracture, or severe aortic calcification. The lumbar spine BMD was measured for 30 patients, and the total hip BMD for four patients (two men and two postmenopausal women).

\section{Statistical analysis}

Data were expressed as the mean \pm standard deviation. A one-way analysis of variance (ANOVA) with repeated measurements was used to determine the significance of longitudinal changes in the BMD and biochemical markers.
A two-way ANOVA with repeated measurements was used to compare longitudinal changes in the BMD and biochemical markers between men and postmenopausal women. A Fisher's exact test was used to compare the incidence of fractures between men and postmenopausal women. All the statistical analyses were performed using the Stat View-J5.0 (Abacus Concepts Inc, Berkeley, CA) program on a Windows ${ }^{\circledR}$ computer. A significance level of $P<0.05$ was used for all the comparisons.

\section{Results \\ Characteristics of the study subjects at the start of treatment}

Six patients were men and 28 patients were postmenopausal women. Twelve patients had diseases that might affect bone metabolism: glucocorticoid use due to asthma or subacute thyroiditis $(n=4)$, hyperthyroidism $(n=2)$, gastrectomy $(n=2)$, breast cancer-induced bone loss due to aromatase inhibitors $(\mathrm{n}=1)$, warfarin used after valve transplantation of the heart $(n=1)$, osteogenesis imperfecta $(n=1)$, and Parkinson's disease $(n=1)$. Table 1 shows the characteristics of the study subjects at the start of the treatment. The mean age of all the subjects was 67.4 years (range: $41-80$ years). The mean percentage of the YAM in the lumbar spine and total hip BMD was $45.8 \%$ and $43.8 \%$, respectively. The mean levels of serum calcium, phosphorus, and ALP were

Table I Characteristics of study subjects at the start of treatment

\begin{tabular}{|c|c|}
\hline Characteristic & Value \\
\hline Gender (male/female) & $6 / 28$ \\
\hline Age (years) & $67.4 \pm 9.5(4 I-80)$ \\
\hline Height (m) & $1.50 \pm 0.10(1.3 \mathrm{I}-1.77)$ \\
\hline Body weight (kg) & $45.8 \pm 8.8(35-68)$ \\
\hline Body mass index $\left(\mathrm{kg} / \mathrm{m}^{2}\right)$ & $20.3 \pm 2.2(16.8-26.7)$ \\
\hline Lumbar spine BMD (g/cm²) & $0.464 \pm 0.042(0.323-0.511)$ \\
\hline \%YAM of lumbar spine BMD (\%) & $45.8 \pm 4.0(33-50)$ \\
\hline Total hip BMD $\left(\mathrm{g} / \mathrm{cm}^{2}\right)$ & $0.395 \pm 0.07$ I $(0.293-0.450)$ \\
\hline \%YAM of total hip BMD (\%) & $43.8 \pm 5.2(36-47)$ \\
\hline Serum calcium $(\mathrm{mg} / \mathrm{dL})$ & $9.4 \pm 0.4(8.7-10.2)$ \\
\hline Serum phosphorus (mg/dL) & $3.2 \pm 0.4(2.4-4.0)$ \\
\hline Serum ALP (IU/L) & $297 \pm 113(133-532)$ \\
\hline Urinary NTX (nmol BCE/mmol Cr) & $84.9 \pm 39.4(42.8-205.3)$ \\
\hline $\begin{array}{l}\text { Number }(\%) \text { of patients with prevalent } \\
\text { vertebral fractures }\end{array}$ & $30(88.2 \%)$ \\
\hline $\begin{array}{l}\text { Number }(\%) \text { of patients with history of } \\
\text { non-vertebral fractures }\end{array}$ & $6(17.6 \%)$ \\
\hline
\end{tabular}

Notes: Data are expressed as means \pm SD. Numbers in the parenthesis of the right column are ranges.

Abbreviations: BMD, bone mineral density; YAM, young adult mean; ALP, alkaline phosphatase; NTX, cross linked N-terminal telopeptides of type I collagen; BCE, bone collagen equivalent; $\mathrm{Cr}$, creatinine. 
within the normal ranges $(8.4-10.2 \mathrm{mg} / \mathrm{dL}, 2.5-4.5 \mathrm{mg} / \mathrm{dL}$, and 100-340 IU/L, respectively). The mean level of urinary NTX was higher than the normal range for Japanese women (9.3-54.3 nmol BCE/mmol Cr), ${ }^{24}$ indicating a high turnover characteristic of osteoporosis. All patients had osteoporotic vertebral or nonvertebral (hip, distal radius, and proximal humerus) fractures. The number of women with prevalent vertebral fractures was $30(88.2 \%)$, and the number of patients with a history of nonvertebral fractures was six (17.6\%).

\section{Changes in lumbar spine and total hip BMD}

Figure 1 shows that the lumbar spine BMD continued to increase for 3 years. A one-way ANOVA with repeated measurements showed significant longitudinal changes in the lumbar spine BMD $(P<0.0001)$. The mean rates of change in the lumbar spine BMD after 1, 2, and 3 years of treatment were $+11.3 \%,+12.4 \%$, and $+14.8 \%$, respectively. However, total hip BMD did not change significantly $(P=0.8706)$. The mean rates of change in the total hip BMD after 1,2 , and 3 years of treatment were $-0.5 \%,+3.2 \%$, and $+6.4 \%$, respectively.

A two-way ANOVA with repeated measurements showed that longitudinal changes in the BMD did not differ significantly between men and postmenopausal women $(P=0.8423$ for the lumbar spine and $P=0.1971$ for the total hip).

\section{Changes in biochemical markers}

Figure 2 shows the changes in the biochemical markers. The mean urinary NTX level decreased to the normal range

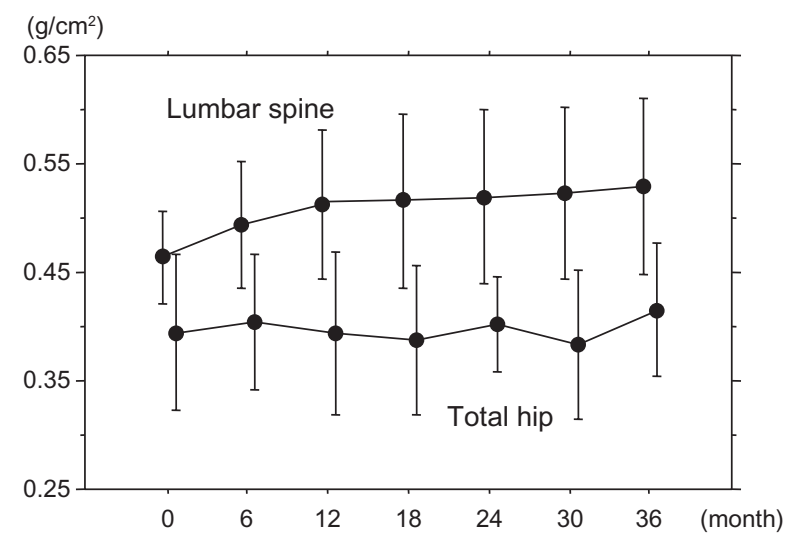

Figure I Changes in lumbar spine and total hip BMD.

Notes: Data are expressed as the mean \pm SD. A one-way ANOVA with repeated measurements showed significant longitudinal changes in the lumbar spine BMD $(P<0.000 \mathrm{I})$. The mean rates of change in the lumbar spine BMD after I, 2, and 3 years of treatment were $+11.3 \%,+12.4 \%$, and $+14.8 \%$, respectively. However, total hip BMD did not change significantly $(P=0.8706)$. The mean rates of change in the total hip BMD after I, 2 , and 3 years of treatment were $-0.5 \%,+3.2 \%$, and $+6.4 \%$, respectively.

Abbreviations: ANOVA, analysis of variance; BMD, bone mineral density. for Japanese women $(9.3-54.3 \mathrm{nmol} \mathrm{BCE} / \mathrm{mmol} \mathrm{Cr})^{24}$ after 3 months of treatment, and the mean serum ALP level decreased but remained within the normal range (135-310 IU/L) during the 3-year period. A one-way ANOVA with repeated measurements showed significant longitudinal changes in the serum ALP and urinary NTX levels (both $P<0.0001)$. The mean rates of change in the urinary NTX level after 3 months of treatment were $-42.5 \%$. The mean rates of change in the serum ALP level after 1, 2, and 3 years of treatment were $-26.1 \%,-20.5 \%$, and $-18.9 \%$, respectively. However, the serum calcium and phosphorus levels did not change significantly ( $P=0.0760$ and 0.8799 , respectively).

A two-way ANOVA with repeated measurements showed that longitudinal changes in the serum calcium, phosphorus, and ALP and urinary NTX levels did not differ significantly between men and postmenopausal women $(P=0.1832$ for calcium, $P=0.9447$ for phosphorus, $P=0.3251$ for ALP, and $P=0.4121$ for urinary NTX).

\section{Incidence of osteoporotic fractures}

Table 2 shows that during the 3 -year treatment period, nine patients $(26.5 \%)$ experienced vertebral fractures (morphometric fractures in seven patients [20.6\%] and clinical fractures in two patients [5.9\%]). One patient (2.9\%) experienced nonvertebral fracture (proximal humerus fracture).

A Fisher's exact test showed that the incidence of fractures did not differ significantly between men and postmenopausal women (16.7\% for men and 32.1\% for postmenopausal women, $P=0.4502)$.

\section{Adverse events}

No serious adverse events, including osteonecrosis of the jaw, femoral diaphysis atypical fractures, and atrial fibrillation, ${ }^{25-35}$ were observed.

\section{Discussion}

Combined treatment with ALN and ALF increased the lumbar spine BMD $(+11.3 \%,+12.4 \%$, and $+14.8 \%$ at 1,2 , and 3 years, respectively) subsequent to reductions in the urinary NTX level (-42.5\% at 3 months) and the serum ALP level $(-26.1 \%,-20.5 \%$, and $-18.9 \%$ at 1,2 , and 3 years, respectively). RCTs have shown that ALN treatment ( $5 \mathrm{mg}$ daily or $35 \mathrm{mg}$ weekly) decreases the urinary NTX $(-45 \%$ at 3 months) and serum ALP (about $-20 \%$ at 3 years) levels and increases the lumbar spine BMD $(+9.2 \%$ at 3 years $)$ in postmenopausal Japanese women with osteoporosis. ${ }^{8,10}$ Our previous two studies also showed that ALN treatment 

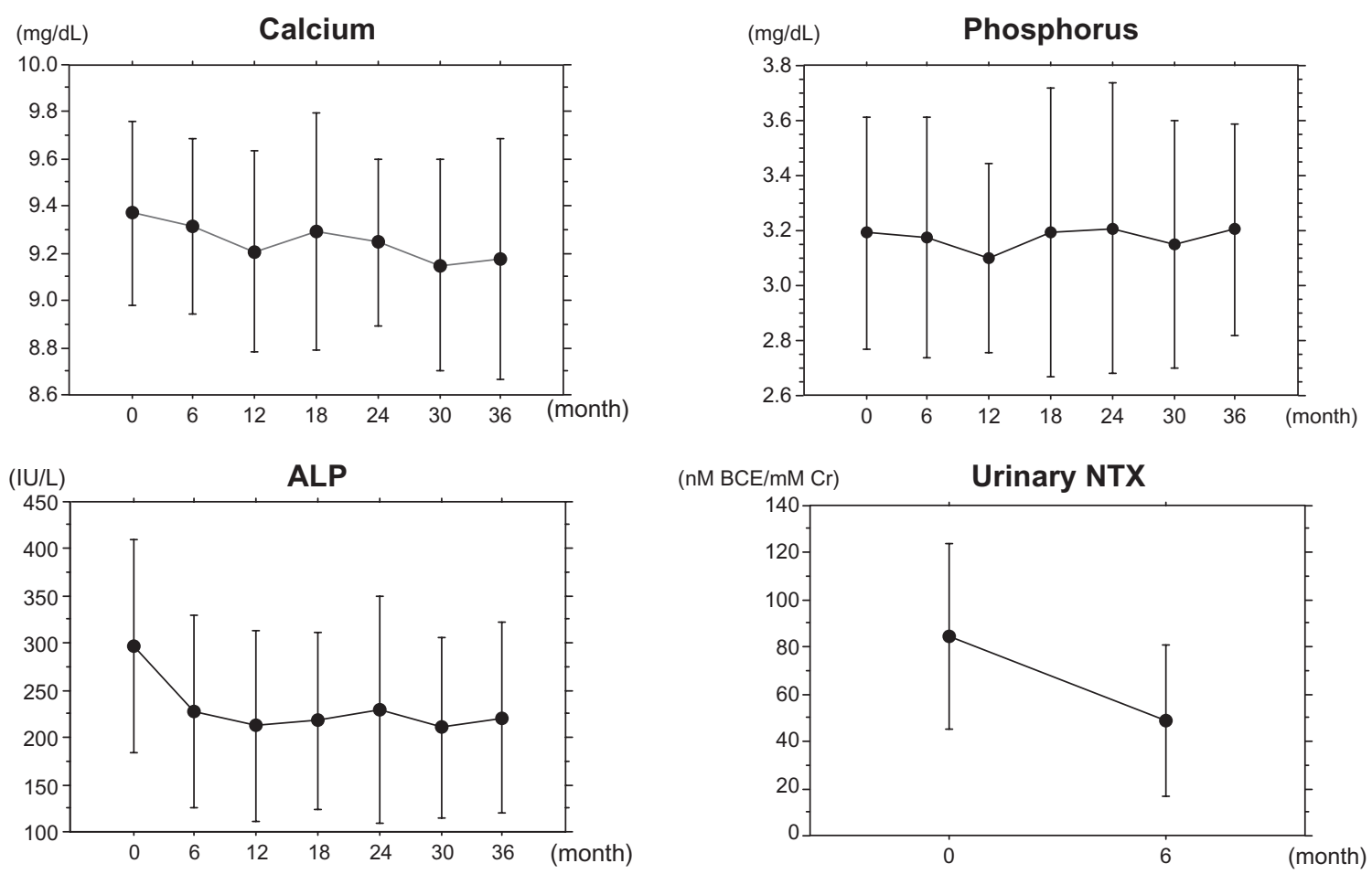

Figure 2 Changes in biochemical markers.

Notes: Data are expressed as the mean \pm SD. A one-way ANOVA with repeated measurements showed significant longitudinal changes in the serum ALP and urinary NTX levels (both $P<0.000$ I). The mean rates of change in the urinary NTX levels after 3 months of treatment were $-42.5 \%$. The mean rates of change in the serum ALP levels after I, 2 , and 3 years of treatment were $-26.1 \%,-20.5 \%$, and $-18.9 \%$, respectively. However, the serum calcium and phosphorus levels did not change significantly $(P=0.0760$ and 0.8799 , respectively).

Abbreviations: ANOVA, analysis of variance; ALP, alkaline phosphatase; NTX, cross linked N-terminal telopeptides of type I collagen; BCE, bone collagen equivalent; Cr, creatinine.

in postmenopausal women with osteoporosis and men with osteoporosis increased the lumbar spine BMD $(+5.8 \%$ at 1 year and $+9.4 \%$ at 3 years, respectively) subsequent to reductions in the urinary NTX level $(-39.2 \%$ and $-40.0 \%$, respectively, at 3 months) and the serum ALP level ( $-17.8 \%$ at 1 year and $-21.2 \%$ at 3 years, respectively). ${ }^{36,37}$ On the other hand, an RCT showed that ALF mildly increased the lumbar spine BMD (1.35\% at 1 year) without significantly influencing bone turnover markers in postmenopausal women with osteoporosis. ${ }^{9}$ The increase in the lumbar spine BMD in the present study appears to be greater than those in the previous studies regarding ALN treatment, despite the similar reductions in bone turnover markers,

Table 2 Incidence of vertebral and nonvertebral fractures

\begin{tabular}{ll}
\hline Fracture & Incidence $\mathbf{n}(\%)$ \\
\hline Vertebral & \\
Morphometric & $7(20.6 \%)$ \\
Clinical & $2(5.9 \%)$ \\
Nonvertebral & \\
Hip & $0(0.0 \%)$ \\
Distal radius & $0(0.0 \%)$ \\
Proximal humerus & $1(2.9 \%)$ \\
\hline
\end{tabular}

suggesting the additive effect of ALN and ALF on the lumbar spine BMD.

The results of the Fracture Intervention Trial revealed that ALN effectively reduced the fracture risk in postmenopausal women with vertebral fractures and low BMD, including those women at the highest risk because of advanced age or severe osteoporosis. ${ }^{38}$ Although the relative risk for vertebral fracture was compared between age $<75$ years and $\geq 75$ years, femoral neck BMD $<0.59 \mathrm{~g} / \mathrm{cm}^{2}$ and $\geq 0.59 \mathrm{~g} / \mathrm{cm}^{2}$, one existing vertebral fracture and $\geq 2$ existing vertebral fractures, or history of postmenopausal fracture and no history of postmenopausal fracture in this analysis, the incidence of vertebral fractures after ALN treatment was not compared among groups.

The incidence of vertebral fractures was $26.5 \%$ (morphometric fracture $20.6 \%$ and clinical fracture $5.9 \%$ ) in patients (mean age: 67.8 years) with severe bone loss (100\% with a history of osteoporotic fracture). Our previous study revealed that the incidence of vertebral fractures after 3-year ALN treatment was 13.3\% (morphometric fracture $9.6 \%$ and clinical fracture $3.7 \%$ ) in postmenopausal women (mean age: $67.4 \%$ ) with osteoporosis $(37 \%$ 
with a history of osteoporotic fracture). ${ }^{36}$ Thus, despite the greater increase in the lumbar spine BMD, the incidence of vertebral fractures was considered to be higher in patients with severe bone loss and osteoporotic fracture than those with postmenopausal osteoporosis. This result suggests the importance of BMD and preexisting osteoporotic fractures at baseline in the prevention of vertebral fractures by bisphosphonate treatment. Shiraki et $\mathrm{al}^{39}$ reported that traditional risk factors such as older age, low BMD, and preexisting osteoporotic fractures were recognized as risks for incident fractures in postmenopausal osteoporotic women treated with bisphosphonates, supporting our suggestion. Thus, anabolic agents, such as teriparatide, which are able to vigorously increase lumbar spine $\mathrm{BMD}^{40}$ should be considered as the first-line drugs in the treatment of severe osteoporosis.

The total hip BMD did not change significantly from the baseline values in four patients (two men and two postmenopausal women). Our previous study showed that 1-year treatment with ALN increased the total hip BMD by $2.3 \%$ from the baseline values in men with osteoporosis or osteopenia and clinical risk factors of fractures. ${ }^{14}$ However, the effect of ALN on total hip BMD has been less established in postmenopausal Japanese women with osteoporosis. In the present study, the mean rates of change in total hip BMD after 1, 2, and 3 years of treatment were $-0.5 \%,+3.2 \%$, and $+6.4 \%$, respectively, which was not statistically significant. This result may be attributable to the small sample size. Further studies are needed to establish the effect of ALN treatment with or without ALF on the total hip BMD in men and postmenopausal women with osteoporosis in Japan.

A couple of German studies showed that combined treatment with ALN and ALF improved physical function (chair rising time and time up and go) and back pain in elderly patients with a high risk of falls and fractures. ${ }^{41,42}$ Although we did not assess the risk of falls and back pain, it would be of interest to investigate the effect of combined treatment with ALN and ALF on fall incidence and subsequent fractures in frail patients with severe osteoporosis.

An epidemiological study has indicated that the incidence of vertebral fractures is higher and that of hip fractures is lower among elderly Japanese people than among elderly Western people. ${ }^{6}$ In the present study, no patients suffered from distal radius or hip fractures, although the incidence of vertebral fractures was considered to be high. Vitamin D supplementation is linked to a decreased risk of falls in ambulatory and institutionalized elderly patients, because muscle tissue has highly specific nuclear receptors for 1,25-dihydroxyvitamin D. ${ }^{43}$ Bischoff-Ferrari et al. ${ }^{44,45}$ have shown that vitamin D supplementation appears to reduce the risk of falls and hip and any non-vertebral fractures in ambulatory or institutionalized elderly persons. Thus, ALF might have played a significant role in reducing the risk of falls and subsequent fractures in the present study.

There is a notable limitation. Because the present study was a retrospective study with a small sample size, the results might be biased. RCTs are needed to confirm the effect of combined treatment with ALN and ALF on severe osteoporosis.

In conclusion, the present retrospective study showed that combined treatment with ALN and ALF suppressed bone turnover and increased lumbar spine BMD, compared with baseline values, over the course of a 3-year treatment period without causing any severe adverse events in patients with severe bone loss and osteoporotic fracture. However, its efficacy against vertebral fractures may not be sufficient. Thus, anabolic agents such as teriparatide should be taken into consideration as first-line drugs in patients with severe osteoporosis.

\section{Disclosure}

The authors report no funding sources or conflicts of interest in this work.

\section{References}

1. Black DM, Cummings SR, Karpf DB, et al. Randomised trial of effect of alendronate on risk of fracture in women with existing vertebral fractures. Lancet. 1996;348:1535-1541.

2. Cummings SR, Black DM, Thompson DE, et al. Effect of alendronate on risk of fracture in women with low bone density but without vertebral fractures: results from the Fracture Intervention Trial. JAMA. 1998; 280:2077-2082.

3. Wells GA, Cranney A, Peterson J, et al. Alendronate for the primary and secondary prevention of osteoporotic fractures in postmenopausal women. Cochrane Database Syst Rev. 2008;23(1):CD001155.

4. Adachi JD, Saag KG, Delmas PD, et al. Two-year effects of alendronate on bone mineral density and vertebral fracture in patients receiving glucocorticoids: a randomized, double-blind, placebo-controlled extension trial. Arthritis Rheum. 2001;44:202-211.

5. Ringe JD, Dorst A, Faber H, et al. Alendronate treatment of established primary osteoporosis in men: 3-year results of a prospective, comparative, two-arm study. Rheumatol Int. 2004;24:110-113.

6. Fujiwara S, Kasagi F, Masunari N, et al. Fracture prediction from bone mineral density in Japanese men and women. J Bone Miner Res. 2003; 18:1547-1553.

7. Kushida K, Shiraki M, Nakamura T, et al. The efficacy of alendronate in reducing the risk of vertebral fracture in Japanese patients with osteoporosis: a randomized, double-blind, active-controlled, double-dummy trial. Curr Ther Res Clin Exp. 2002;63:606-620. 
8. Kushida K, Shiraki M, Nakamura T, et al. Alendronate reduced vertebral fracture risk in postmenopausal Japanese women with osteoporosis: a 3-year follow-up study. J Bone Miner Metab.2004;22: 462-468.

9. Shiraki M, Kushida K, Fukunaga M, et al. A double-masked multicenter comparative study between alendronate and alfacalcidol in Japanese patients with osteoporosis. Osteoporos Int. 1999;10:183-192.

10. Uchida S, Taniguchi T, Shimizu T, et al. Therapeutic effects of alendronate $35 \mathrm{mg}$ once weekly and $5 \mathrm{mg}$ once daily in Japanese patients with osteoporosis: a double-blind, randomized study. J Bone Miner Metab. 2005;23:382-388.

11. Kitazaki S, Mitsuyama K, Masuda J, et al. Clinical trial: comparison of alendronate and alfacalcidol in glucocorticoid-associated osteoporosis in patients with ulcerative colitis. Aliment Pharmacol Ther. 2009 29:424-430.

12. Okada Y, Nawata M, Nakayamada S, et al. Alendronate protects premenopausal women from bone loss and fracture associated with high-dose glucocorticoid therapy. J Rheumatol. 2008;35: 2249-2254.

13. Takeda S, Kaneoka H, Saito T. Effect of alendronate on glucocorticoidinduced osteoporosis in Japanese women with systemic autoimmune diseases: versus alfacalcidol. Mod Rheumatol. 2008;18:271-276.

14. Iwamoto J, Sato Y, Uzawa M, et al. Comparison of the effects of alendronate and alfacalcidol on hip bone mineral density and bone turnover in Japanese men having osteoporosis or osteopenia with clinical risk factors for fractures. Yonsei Med J. 2009;50:474-481.

15. Orimo H, Sugioka Y, Fukunaga M, et al. Diagnostic criteria of primary osteoporosis. J Bone Miner Metab. 1998;16:139-150.

16. Orimo H, Hayashi Y, Fukunaga M, et al; for the Osteoporosis Diagnostic Criteria Review Committee, Japanese Society for Bone and Mineral Research. Diagnostic criteria for primary osteoporosis: year 2000 revision. J Bone Miner Metab. 2001;19:331-337.

17. Iwamoto J, Miyata A, Sato Y, et al. Five-year alendronate treatment outcome in older postmenopausal Japanese women with osteoporosis or osteopenia and clinical risk factors for fractures. Ther Clin Risk Manag. 2009;5:773-779.

18. Iwamoto J, Sato Y, Uzawa M, et al. Seven years' experience with alendronate in postmenopausal Japanese women with osteoporosis Ther Clin Risk Manag. 2010;6:201-206.

19. Iwamoto J, Sato Y, Uzawa M, et al. Experience with alendronate treatment for four years among Japanese men with osteoporosis or osteopenia and clinical risk factors for fractures. Ther Clin Risk Manag 2010;6:593-600.

20. Ringe JD, Farahmand P, Schacht E, et al. Superiority of a combined treatment of alendronate and alfacalcidol compared to the combination of alendronate and plain vitamin d or alfacalcidol alone in established postmenopausal or male osteoporosis (AAC-Trial). Rheumatol Int. 2007;27:425-434.

21. Felsenberg D, Bock O, Börst H, et al. Additive impact of alfacalcidol on bone mineral density and bone strength in alendronate treated postmenopausal women with reduced bone mass. J Musculoskelet Neuronal Interact. 2011;11:34-45.

22. Orimo H, Shiraki M, Hayashi Y, et al. Effects of 1 alpha-hydroxyvitamin $\mathrm{D}_{3}$ on lumbar bone mineral density and vertebral fractures in patients with postmenopausal osteoporosis. Calcif Tissue Int. 1994;54:370-376.

23. Iwamoto J, Takeda T, Sato Y, et al. Early changes in urinary cross-linked $\mathrm{N}$-terminal telopeptides of type I collagen level correlate with one-year response of lumbar bone mineral density to alendronate in Japanese postmenopausal women with osteoporosis. J Bone Miner Metab. 2005;23:238-242.

24. Nishizawa Y, Nakamura T, Ohta H, et al; for the Committee on the Guidelines for the Use of Biochemical Markers of Bone Turnover in Osteoporosis Japan Osteoporosis Society. Guidelines for the use of biochemical markers of bone turnover in osteoporosis (2004). J Bone Miner Metab. 2005;23:97-104.
25. Ruggiero SL, Dodson TB, Assael LA, et al; for the American Association of Oral and Maxillofacial Surgeons. American Association of Oral and Maxillofacial Surgeons position paper on bisphosphonate-related osteonecrosis of the jaws - 2009 update. J Oral Maxillofac Surg. 2009;67:2-12.

26. Lo JC, O'Ryan FS, Gordon NP, et al; for the Predicting Risk of Osteonecrosis of the Jaw with Oral Bisphosphonate Exposure (PROBE) Investigators. Prevalence of osteonecrosis of the jaw in patients with oral bisphosphonate exposure. J Oral Maxillofac Surg. 2010;68: 243-253.

27. Lenart BA, Lorich DG, Lane JM. Atypical fractures of the femoral diaphysis in postmenopausal women taking alendronate. $N$ Engl J Med. 2008;358:1304-1306.

28. Rizzoli R, Akesson K, Bouxsein M, et al. Subtrochanteric fractures after long-term treatment with bisphosphonates: a European Society on Clinical and Economic Aspects of Osteoporosis and Osteoarthritis, and International Osteoporosis Foundation Working Group Report. Osteoporos Int. 2011;22:373-390.

29. Giusti A, Hamdy NA, Papapoulos SE. Atypical fractures of the femur and bisphosphonate therapy: a systematic review of case/case series studies. Bone. 2010;47:169-180.

30. Giusti A, Hamdy NA, Dekkers OM, et al. Atypical fractures and bisphosphonate therapy: a cohort study of patients with femoral fracture with radiographic adjudication of fracture site and features. Bone. 2011 48(5):966-971.

31. Shane E, Burr D, Ebeling PR, et al; for the American Society for Bone and Mineral Research. Atypical subtrochanteric and diaphyseal femoral fractures: report of a task force of the American Society for Bone and Mineral Research. J Bone Miner Res. 2010;25: 2267-2294

32. Pazianas M, Compston J, Huang CL. Atrial fibrillation and bisphosphonate therapy. J Bone Miner Res. 2010;25:2-10.

33. Heckbert SR, Li G, Cummings SR, et al. Use of alendronate and risk of incident atrial fibrillation in women. Arch Intern Med. 2008;168: 826-831.

34. Pazianas M, Cooper C, Ebetino FH, et al. Long-term treatment with bisphosphonates and their safety in postmenopausal osteoporosis. Ther Clin Risk Manag. 2010;6:325-343.

35. Vestergaard P, Schwartz K, Pinholt EM, et al. Risk of atrial fibrillation associated with use of bisphosphonates and other drugs against osteoporosis: a cohort study. Calcif Tissue Int. 2010;86:335-342.

36. Iwamoto J, Takeda T, Sato Y, et al. Comparison of the effect of alendronate on lumbar bone mineral density and bone turnover in men and postmenopausal women with osteoporosis. Clin Rheumatol. 2007; 26:161-167.

37. Iwamoto J, Sato Y, Uzawa M, et al. Three-year experience with alendronate treatment in postmenopausal osteoporotic Japanese women with or without type 2 diabetes. Diabetes Res Clin Practice. Epub 2011 Apr 25.

38. Ensrud KE, Black DM, Palermo L, et al. Treatment with alendronate prevents fractures in women at highest risk: results from the Fracture Intervention Trial. Arch Intern Med. 1997;157:2617-2624.

39. Shiraki M, Yamazaki Y, Shiraki Y, et al. High level of serum undercarboxylated osteocalcin in patients with incident fractures during bisphosphonate treatment. J Bone Miner Metab. 2010;28: 578-584.

40. Miyauchi A, Matsumoto T, Sugimoto T, et al. Effects of teriparatide on bone mineral density and bone turnover markers in Japanese subjects with osteoporosis at high risk of fracture in a 24-month clinical study: 12-month, randomized, placebo-controlled, double-blind and 12-month open-label phases. Bone. 2010;47:493-502.

41. Ringe JD, Schacht E, Dukas L, et al. Potency of a combined alfacalcidol-alendronate therapy to reduce the risk of falls and fractures in elderly patients with glucocorticoid-induced osteoporosis. Arzneimittelforschung. 2011;61:104-111. 
42. Schacht E, Ringe JD. Risk reduction of falls and fractures, reduction of back pain and safety in elderly high risk patients receiving combined therapy with alfacalcidol and alendronate: a prospective study. Arzneimittelforschung. 2011;61:40-54.

43. Bischoff HA, Borchers M, Guadt F, et al. In situ detection of 1,25-dihydroxyvitamin D3 receptor in human skeletal muscle tissue. Histochem J. 2001;33:19-24.
44. Bischoff-Ferrari HA, Dawson-Hughes B, Willett WC, et al. Effect of vitamin D on falls: a meta-analysis. JAMA. 2004;291:1999-2006.

45. Bischoff-Ferrari HA, Willett WC, Wong JB, et al. Fracture prevention with vitamin D supplementation: a meta-analysis of randomized controlled trials. JAMA. 2005;293:2257-2264.

\section{Publish your work in this journal}

Therapeutics and Clinical Risk Management is an international, peerreviewed journal of clinical therapeutics and risk management, focusing on concise rapid reporting of clinical studies in all therapeutic areas, outcomes, safety, and programs for the effective, safe, and sustained use of medicines. This journal is indexed on PubMed Central, CAS,
EMBase, Scopus and the Elsevier Bibliographic databases. The manuscript management system is completely online and includes a very quick and fair peer-review system, which is all easy to use. Visit http://www.dovepress.com/testimonials.php to read real quotes from published authors.

Submit your manuscript here: http://www.dovepress.com/therapeutics-and-clinical-risk-management-journal 